\title{
Inorganic Iron Effects on In Vitro Hypoxic Proximal Renal Tubular Cell Injury
}

Richard A. Zager, Brian A. Schimpf, Charles R. Bredl, and Dennis J. Gmur

Department of Medicine, University of Washington, Seattle, Washington 98195; and the Fred Hutchinson

Cancer Research Center, Seattle, Washington 98104

\begin{abstract}
Iron-dependent free radical reactions and renal ischemia are believed to be critical mediators of myohemoglobinuric acute renal failure. Thus, this study assessed whether catalytic iron exacerbates $\mathrm{O}_{2}$ deprivation-induced proximal tubular injury, thereby providing an insight into this form of renal failure. Isolated rat proximal tubular segments (PTS) were subjected to either hypoxia/reoxygenation (H/R: 27:15 min), "chemical anoxia" (antimycin $A ; 7.5 \mu M \times 45$ min), or continuous oxygenated incubation \pm ferrous $\left(\mathrm{Fe}^{2+}\right)$ or ferric $\left(\mathrm{Fe}^{3+}\right)$ iron addition. Cell injury (\% lactic dehydrogenase $[\mathrm{LDH}]$ release ), lipid peroxidation (malondialdehyde, [MDA]), and ATP depletion were assessed. Under oxygenated conditions, $\mathrm{Fe}^{2+}$ and $\mathrm{Fe}^{3+}$ each raised MDA $(\sim 7-10 \times)$ and decreased ATP $(\sim 25 \%)$. $\mathrm{Fe}^{2+}$, but not $\mathrm{Fe}^{3+}$, caused LDH release (31 $\pm 2 \%$ ). During hypoxia, $\mathrm{Fe}^{2+}$ and $\mathrm{Fe}^{3+}$ worsened ATP depletion; however, each decreased $\mathrm{LDH}$ release $(\sim 31$ to $\sim 22 \% ; P<0.01)$. $\mathrm{Fe}^{2+}$-mediated protection was negated during reoxygenation because $\mathrm{Fe}^{2+}$ exerted its intrinsic cytotoxic effect ( $\mathrm{LDH}$ release: $\mathrm{Fe}^{2+}$ alone, 31 $\pm 2 \%$; H / R 36 $\pm 2 \%$; H R $+\mathrm{Fe}^{2+}, 41 \pm 2 \%$ ). However, $\mathrm{Fe}^{3+}$-mediated protection persisted throughout reoxygenation because it induced no direct cytotoxicity $(H / R, 39 \pm 2 \% ; H / R$ $\left.+\mathrm{Fe}^{3+}, 25 \pm 2 \% ; P<0.002\right) . \mathrm{Fe}^{3+}$ also decreased antimycin toxicity ( $41 \pm 4$ vs. $25 \pm 3 \% ; P<0.001)$ despite inducing marked lipid peroxidation and without affecting ATP. These results indicate that catalytic iron can mitigate, rather than exacerbate, $\mathrm{O}_{2}$ deprivation/reoxygenation PTS injury. (J. Clin. Invest. 1993. 91:702-708.) Key words: adenosine triphosphate • antimycin $\mathbf{A} \cdot$ hypoxia $\bullet$ iron $\bullet$ malondialdehyde
\end{abstract}

\section{Introduction}

For almost 50 years it has been recognized that myohemoglobinuria and renal hypoperfusion/ischemia cause synergistic tubular injury, potentially triggering severe acute renal failure $(\mathrm{ARF})^{1}(1,2)$. However, the mechanism(s) by which they do so have remained poorly defined. Three general possibilities have been considered, focusing at the vascular, intraluminal,

Address reprint requests to Dr. Richard A. Zager, Nephrology Department, M-178, Fred Hutchinson Cancer Research Center, 1124 Columbia Street, Seattle, WA 98104.

Received for publication 1 June 1992 and in revised form 18 September 1992.

1. Abbreviations used in this paper: ARF, acute renal failure; DFO, deferoxamine; $\mathrm{LDH}$, lactic dehydrogenase; MDA, malondialdehyde; PTS, isolated proximal tubular segments.

J. Clin. Invest.

(C) The American Society for Clinical Investigation, Inc.

0021-9738/93/02/0702/07 \$2.00

Volume 91, February 1993, 702-708 and tubular cell levels. First, since heme proteins can lower renal blood flow (e.g., references 3 and 4), myohemoglobinuria may exacerbate tubular cell ATP decrements during a superimposed hypotensive/renal vasoconstrictive event. Second, since heme proteins form intraluminal casts under aciduric conditions, a worsening of ischemia-induced intratubular obstruction may result, potentiating filtration failure $(5,6)$. Third, heme protein endocytosis renders proximal tubular cells highly vulnerable to superimposed ischemic insults (6). Since proximal tubular necrosis is a critical determinant of both heme protein and ischemic ARF, synergistic tubular injury is undoubtedly an important underlying mechanism for the heme protein-ischemic interaction.

Given that heme proteins and ischemia can each induce injury at the vascular, intraluminal, and tubular cell levels, and because secondary interactions can then occur, it has been difficult to dissect out direct mechanisms of synergistic tubular cell injury in whole-animal experiments. For example, pharmacological probes used to study cellular mechanisms of injury (e.g., mannitol, deferoxamine) can yield misleading information since they may exert vascular $(7,8)$, intraluminal $(7-10)$, and direct tubular cell effects $(7,10-13)$. An additional complexity stemming from in vivo experiments is that heme proteins, which are not directly cytotoxic (e.g., references 14 and 15 ), probably need to be "processed" to induce their adverse effects. For example, several in vivo (11-13) and in vitro (10, 15 ) studies strongly suggest that free iron, released from heme proteins within the circulation (10) and possibly within the kidney, is partially responsible for heme protein cytotoxicity. Thus, a given in vivo intervention might confer cytoprotection by affecting free iron availability (10) without providing direct insights into mechanisms of tubular injury.

In an attempt to circumvent some of these problems, the present study was undertaken to ascertain direct iron effects on $\mathrm{O}_{2}$ deprivation/reoxygenation injury of isolated rat proximal tubular segments (PTSs). Since neither vascular effects nor cast formation are operative in the PTS system, and because a constant amount of iron can be delivered, it was reasoned that these in vitro experiments might yield new mechanistic insights into the nature of the heme protein/iron-ischemic interaction. To this end, the following specific questions were addressed: Does free $\mathrm{Fe}^{2+}$, by itself cytotoxic (15), render proximal tubular cells highly vulnerable to superimposed $\mathrm{O}_{2}$ deprivation/ reoxygenation injury? Since iron is capable of catalyzing oxygen-based free radical reactions $(16,17)$, does an exogenous iron challenge drive hypoxic/reoxygenation proximal tubular oxidant stress? Alternatively, does $\mathrm{O}_{2}$ deprivation limit the ability of iron to promote oxygen-based free radical reactions? and What effect does free iron have on tubular cell energy levels in the presence and absence of superimposed $\mathrm{O}_{2}$ deprivation? The relevance of this last question stems from in vivo observations that myoglobin infusion lowers renal cortical ATP concentrations, that these ATP decrements are completely blocked by iron chelation (deferoxamine) therapy, and that this iron-me- 
diated effect is apparently independent of changes in renal hemodynamics (18). Thus, if free iron directly synergizes $\mathrm{O}_{2}$ deprivation-mediated tubular ATP depletion, this would provide a potential new insight into the nature of the in vivo heme protein-ischemic interaction. Investigations into each of these three issues form the basis of this report.

\section{Methods}

PTS preparation. Normal male Sprague-Dawley rats ( $150-250 \mathrm{~g}$; Harlan Sprague Dawley, Inc., Indianapolis, IN), maintained under standard vivarium conditions, were used for all PTS preparations, as previously described $(10,15)$. In brief, the rats were anesthetized with pentobarbital (30-40 mg/ $\mathrm{kg}$ i.p.), the kidneys were removed without in vivo perfusion, and the cortices were resected and minced with a razor blade at $4^{\circ} \mathrm{C}$. The resulting tissues were digested for 30 to $40 \mathrm{~min}$ in a collagenase containing buffer to which $1 \mathrm{mM}$ deferoxamine (DFO) had been added to chelate any free iron generated during the mincing/ digestion process (thereby preventing iron toxicity from occurring before experimentation) (19). Viable PTS were pelleted through a $31 \%$ Percoll gradient, they were washed five times to remove any remaining collagenase and DFO, and then they were suspended to a concentration of 1.5 to $3.0 \mathrm{mg}$ protein $/ \mathrm{ml}$ in a buffer with the following composition (mM): $100 \mathrm{NaCl}, 2.1 \mathrm{KCl}, 25 \mathrm{NaHCO}_{3}, 2.4 \mathrm{KH}_{2} \mathrm{PO}_{4}, 1.2 \mathrm{CaCl}_{2}$, $1.2 \mathrm{MgCl}_{2}, 1.2 \mathrm{MgSO}_{4}, 5$ glucose, $4 \mathrm{Na}$ lactate, $10 \mathrm{Na}$ butyrate, 1 alanine, and dextran $\mathrm{T}_{40} 0.6 \%$ and gassed with $95 \% \mathrm{O}_{2} / 5 \% \mathrm{CO}_{2}$; final $\mathrm{pH}$ 7.40. The PTS preparation (using kidneys from either one or two rats, depending on the weight of the animals and the number of aliquots needed for a given experiment) were rewarmed from $4^{\circ} \mathrm{C}$ (isolation temperature) to $36^{\circ} \mathrm{C}$ over $15 \mathrm{~min}$. As an assessment of baseline PTS viability, a 150- $\mu$ l aliquot was used to determine the percent of lactic dehydrogenase (LDH) released (percent of total LDH in the PTS supernatant after centrifugation), after completion of the rewarming process $(10,15)$.

Effects of cytotoxic $\mathrm{Fe}^{2+}$ on PTS vulnerability to hypoxic-reoxygenation injury. Previous studies have indicated that $\mathrm{Fe}^{2+}$, but not $\mathrm{Fe}^{3+}$, is cytotoxic to PTS (15). Thus, to explore the effects of iron toxicity on hypoxia-reoxygenation injury, five separate PTS preparations were each divided into four aliquots and incubated in a $36^{\circ} \mathrm{C}$ shaking water bath for $42 \mathrm{~min}$ as follows: controls: continuous oxygenated $\left(95 \% \mathrm{O}_{2}\right.$ / $5 \% \mathrm{CO}_{2}$ ) incubation; hypoxic-reoxygenation incubation (27 min of hypoxia [gassing with $95 \% \mathrm{~N}_{2} / 5 \% \mathrm{CO}_{2}$ ] followed by $15 \mathrm{~min}$ of reoxygenation $\left[95 \% \mathrm{O}_{2} / 5 \% \mathrm{CO}_{2}\right]$ ); incubation with $2 \mathrm{mM} \mathrm{FeSO}_{4}$ under oxygenated conditions; and hypoxic-reoxygenation injury, as per aliquot 2 , in the presence of $2 \mathrm{mM} \mathrm{FeSO}_{4}$. At the completion of the 42-min incubations, cell injury was reassessed by determining the percent of $\mathrm{LDH}$ released.

To assess whether $\mathrm{Fe}^{2+}$ effects in the above experiments were predominantly expressed during the hypoxic or the reoxygenation period, eight additional PTS preparations were treated exactly as detailed above except that the \%LDH release was determined after both 27 and $42 \mathrm{~min}$ of each incubation. Hypoxic and reoxygenation injury in aliquots 2 and 4 was gauged by the percent of LDH released during the $0-27$ - and during the 27-42-min time frames, respectively.

Effect of $\mathrm{Fe}^{2+}$ and hypoxic-reoxygenation injury on PTS lipid peroxidation. In four of the above experiments, lipid peroxidation was assessed both after completing the initial 27-min and the final 15-min incubations. To this end, at the time of aliquot sampling for LDH release, an additional $0.25 \mathrm{ml}$ of suspension was removed, and the PTSs recovered by centrifugation in a microfuge, were assayed for malondialdehyde (MDA) by using the thiobarbituric acid method of Uchiyama and Mihara (20), as previously employed in this laboratory (15). Thus, the effects of $\mathrm{Fe}^{2+}$, hypoxia-reoxygenation, and $\mathrm{Fe}^{2+}$ + hypoxia-reoxygenation on lipid peroxidation could be assessed. The MDA values were expressed as $\mathrm{nmol} / \mathrm{mg}$ PTS protein, the latter being determined by the bicinchoninic acid method (Pierce Chemical Co., Rockford, IL).
Effect of $\mathrm{Fe}^{3+}$ on PTS hypoxic-reoxygenation injury and lipid peroxidation. It has previously been demonstrated that, although $\mathrm{Fe}^{3+}$ induces PTS lipid peroxidation, cell killing does not result (15). Thus, the following experiment was performed to ascertain iron effects on hypoxic-reoxygenation injury in the absence of direct iron-mediated cytotoxicity. Five PTS preparations were each divided into four equal aliquots as follows: 42-min oxygenated incubation; 42-min oxygenated incubation in the presence of $2 \mathrm{mM} \mathrm{FeCl}_{3}\left(\mathrm{Fe}^{3+}\right) ; 27-\mathrm{min}$ hypoxic and then 15 min of reoxygenated incubation; and 27-min hypoxic and then 15-min reoxygenated incubation in the presence of $\mathrm{Fe}^{3+}$. At the completion of the incubations, \%LDH release and MDA concentrations were determined as noted above.

Effect of $\mathrm{Fe}^{2+}$ and $\mathrm{Fe}^{3+}$ on PTS adenine nucleotide concentrations under oxygenated and hypoxic conditions. The following experiment assessed whether iron addition causes PTS ATP losses under oxygenated conditions (as suggested by in vivo experiments; reference 18); whether such losses are sufficient to explain $\mathrm{Fe}^{2+}$-mediated cell killing; and whether iron addition alters hypoxia-induced tubular energy depletion. To these ends, five separate PTS preparations were each divided into six aliquots: continuous oxygenated incubation; oxygenated incubation in the presence of $2 \mathrm{mM} \mathrm{Fe}^{2+}$; oxygenated incubation in the presence of $2 \mathrm{mM} \mathrm{Fe}^{3+}$; hypoxic incubation; hypoxic incubation +2 $\mathrm{mM} \mathrm{Fe}{ }^{2+}$; and hypoxic incubation $+2 \mathrm{mM} \mathrm{Fe}^{3+}$. After completing 27 min under these conditions, an 150- $\mu$ l sample was removed for assessing LDH release, DFO (Ciba Pharmaceuticals Company, Summit, NJ) was immediately added to each aliquot (final concentration, $4 \mathrm{mM}$ ), and then the adenine nucleotides were extracted by adding $66.6 \% \mathrm{TCA}$ (final concentration, $6.66 \%$ ). (Note: These three maneuvers [aliquot sampling and DFO and TCA addition] were performed while maintaining continuous hypoxic conditions. DFO was added because in pilot studies it was documented that free iron partially interferes with adenine nucleotide recovery from TCA, a phenomenon that is prevented by DFO addition). The PTS suspensions were vortexed, centrifuged, and the supernatants extracted in freon-trioctylamine to remove TCA (18). The supernatants were analyzed for ATP, ADP, and AMP by HPLC (21), as previously described (18). All values were expressed as $\mathrm{nmol} / \mathrm{mg}$ PTS protein.

Effect of $\mathrm{Fe}^{2+}$ plus DFO on hypoxic-reoxygenation injury. In each of the above experiments, $\mathrm{Fe}^{2+}$ or $\mathrm{Fe}^{3+}$ induced marked lipid peroxidation. To assess whether lipid peroxidation is a critical determinant of iron effects on hypoxic-reoxygenation injury, the following experiment was performed. Four sets of PTS were subjected to the hypoxia-reoxygenation protocol with either buffer only addition (controls), DFO addition ( $4 \mathrm{mM}$ ), or DFO followed immediately by $2 \mathrm{mM} \mathrm{Fe}^{2+}$ addition. Excess DFO relative to the amount of $\mathrm{Fe}^{2+}$ was added to limit lipid peroxidation (15), but potentially leaving iron (possibly in the feroxamine complex ) available to confer a protective effect. After completing the $42-\mathrm{min}$ incubations, \%LDH release and MDA concentrations were determined.

Antimycin A toxicity: $\mathrm{Fe}^{3+}$ effects on PTS cytotoxicity, lipid peroxidation, and adenine nucleotide concentrations. To further assess the ability of iron to confer protection against ATP depletion-mediated PTS injury, $\mathrm{Fe}^{3+}$ effects against "chiemical anoxia" (i.e., mitochondrial inhibition in the presence of $\mathrm{O}_{2}$ ) was assessed. To this end, the influence of the $\mathrm{Fe}^{3+}$ on PTS injury induced by antimycin $\mathrm{A}$, a mitochondrial respiratory chain inhibitor, was determined. Five PTS preparations were used for this study. Each preparation was divided into four aliquots: control incubation; antimycin $A$ incubation, $7.5 \mu \mathrm{M} ; 2 \mathrm{mM}$ $\mathrm{Fe}^{3+}$ addition; and antimycin $+2 \mathrm{mM} \mathrm{Fe}^{3+}$ addition. Antimycin $\mathrm{A}$ (Sigma Chemical Co., St. Louis, MO) was prepared as a $250 \times$ ethanol stock solution, $10 \mu \mathrm{l}$ of the stock being added to aliquots 2 and 4 . To control for possible independent ethanol effects, $10 \mu \mathrm{l}$ of ethanol (no antimycin) was added to aliquots 1 and 3 . After $45 \mathrm{~min}$ of incubation (each conducted in the presence of $95 \% \mathrm{O}_{2} / 5 \% \mathrm{CO}_{2}$ ), \% LDH release, MDA, and adenine nucleotide concentrations were determined.

Calculations and statistics. All values are given as mean \pm SEM. Overall statistical comparisons were performed by one-way analysis of variance for repeated measures. Specific comparisons were made by 
paired Student's $t$ test, the Bonferroni correction being applied for multiple comparisons. The \%LDH release data reported are the absolute values obtained, i.e., without subtraction of the baseline percent of LDH released ( $5 \pm 1 \%$ for all experiments). Statistical significance was judged by a $P$ value of $<0.05$.

\section{Results}

Effects of $\mathrm{Fe}^{2+}$ on PTS viability in the presence and absence of hypoxic-reoxygenation injury. As depicted in Fig. $1 \mathrm{~A}, \mathrm{Fe}^{2+}$ addition to continuously oxygenated PTS caused significant cytotoxicity, $31 \pm 2 \% \mathrm{LDH}$ release being observed after $42 \mathrm{~min}$ of incubation $(P<0.001$ vs. time-matched oxygenated control of $9 \pm 1 \%$ ). Hypoxia-reoxygenation also caused substantial cell killing, $36 \pm 2 \%$ of $\mathrm{LDH}$ being released by the end of the protocol (Fig. $1 A$ ). Despite the fact that $\mathrm{Fe}^{2+}$ and hypoxia-reoxygenation each induced substantial cell killing ( 31 and $36 \%$ LDH release, respectively), the combination of $\mathrm{Fe}^{2+}$ plus hypoxia/ reoxygenation produced only $41 \pm 2 \% \mathrm{LDH}$ release, far less than the sum of their independent effects $(67 \%)$. Of note, a lack of more substantial LDH release in these experiments could not be attributed to a "ceiling" effect (i.e., only $41 \%$ of cells could be killed), since in pilot studies performed at this time it was found that prolonged hypoxia ( $50 \mathrm{~min}$ ) induced 56 , 64 , and $65 \%$ LDH release, in three PTS preparations so tested.

To more fully explore why $\mathrm{Fe}^{2+}$ plus hypoxia-reoxygenation induced far less LDH release than the sum of their independent injurous effects, \%LDH release was assessed after hypoxia without reoxygenation, both in the presence and absence of $\mathrm{Fe}^{2+}$. As depicted in Fig. $1 \mathrm{~B}, \mathrm{Fe}^{2+}+$ hypoxia induced significantly less $\mathrm{LDH}$ release $(24 \pm 3 \%)$ than did hypoxia alone $(31 \pm 3 \% ; P<0.002)$, indicating that $\mathrm{Fe}^{2+}$ mitigated the hypoxic injury phase. This was despite the fact that $\mathrm{Fe}^{2+}$ induced significant cytotoxicity within this 27 -min time frame when maintained under oxygenated conditions ( $19 \pm 2$ vs. $8 \pm 1 \%$ for non-iron-exposed controls).

$\mathrm{Fe}^{2+}$-mediated lipid peroxidation in the presence and absence of hypoxia-reoxygenation. As depicted in Fig. $2 A$, hypoxia in the absence of $\mathrm{Fe}^{2+}$ halved MDA concentrations compared with coincubated oxygenated controls $(0.4 \pm 0.03$ vs. $0.8 \pm 0.01 \mathrm{nmol} / \mathrm{mg}$ protein; $P<0.05) . \mathrm{Fe}^{2+}$, in the presence of $\mathrm{O}_{2}$, raised MDA concentrations to $7.6 \pm 0.1 \mathrm{nmol} / \mathrm{mg}$ protein. In contrast to the non-iron-exposed PTS (in which hypoxia decreased MDA concentrations), in the presence of $\mathrm{Fe}^{2+}$, hypoxia dramatically increased lipid peroxidation, raising MDA values to $12.7 \pm 0.5 \mathrm{nmol} / \mathrm{mg}$ protein $(P<0.01$ vs. iron incubation alone).

As shown in Fig. $2 B$, after.reoxygenation, the previously hypoxic/non-iron-exposed PTS still had significantly lower MDA values than their continuously oxygenated controls $(0.4 \pm 0.04$ vs. $0.6 \pm 0.05 ; P<0.03$ ) (indicating that hypoxiareoxygenation decreased, rather than increased lipid peroxidation ). Comparable MDA values were observed in the $\mathrm{Fe}^{2+}$-exposed PTS aliquots whether they had been subjected to hypoxia-reoxygenation or to continuous oxygenated incubation (indicating that the greater MDA increments observed during $\mathrm{Fe}^{2+} /$ hypoxic incubation vs. $\mathrm{Fe}^{2+} /$ oxygenated incubation were dissipated during the reoxygenation period).

$\mathrm{Fe}^{3+}$ effects on PTS viability and lipid peroxidation in the presence and absence of hypoxia-reoxygenation. As depicted in Fig. $3 A$, incubating PTS with $\mathrm{Fe}^{3+}$ for 42 min under continuously oxygenated conditions induced no cytotoxicity $(9 \pm 1 \%$

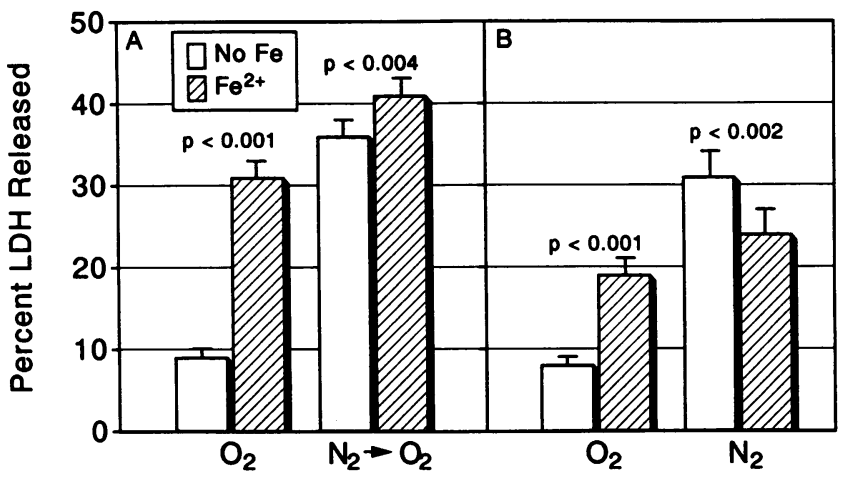

Figure 1. (A) Percent LDH released after either $42 \mathrm{~min}$ of continuous oxygenation $\left(\mathrm{O}_{2}\right)$ or after $27 \mathrm{~min}$ of hypoxia plus $15 \mathrm{~min}$ of reoxygenation $\left(\mathrm{N}_{2} \rightarrow \mathrm{O}_{2}\right)$. Despite the fact that $\mathrm{Fe}^{2+}$ and hypoxia-reoxygenation each caused marked cell injury ( 31 and $36 \%$ LDH release, respectively), together only $41 \% \mathrm{LDH}$ release resulted ( $n=13$ sets of observations). ( $B$ ) Percent LDH released during $27 \mathrm{~min}$ of either oxygenated $\left(\mathrm{O}_{2}\right)$ or hypoxic $\left(\mathrm{N}_{2}\right)$ incubation in the presence or absence of $\mathrm{Fe}^{2+}$. Despite the fact that $\mathrm{Fe}^{2+}$ caused $\mathrm{LDH}$ release under oxygenated conditions ( $P<0.001$ vs. non-iron-exposed controls), it decreased LDH release during hypoxia $(P<0.002$ vs. non-iron-exposed controls; $n=8$ sets of observations).

$\mathrm{LDH}$ release for both $\mathrm{Fe}^{3+}$ and control PTS). $\mathrm{Fe}^{3+}$ exerted a clear cytoprotective effect vs. hypoxic-reoxygenation injury, decreasing \%LDH release from $39 \pm 2$ to $25 \pm 2 \%$ by the end of the 42 -min protocol $(P<0.002)$. To more accurately reflect the degree to which $\mathrm{Fe}^{3+}$ had mitigated hypoxia-reoxygenation-induced cell killing, the background cell injury (\%LDH released after $42 \mathrm{~min}$ of oxygenated incubation; $9 \pm 1 \%$ ) was subtracted from the total \%LDH released after hypoxia-reoxygenation in the absence $(39 \pm 2 \%)$ and presence $(25 \pm 2 \%)$ of $\mathrm{Fe}^{3+}$. By so doing, hypoxic-reoxygenation-specific cell injury

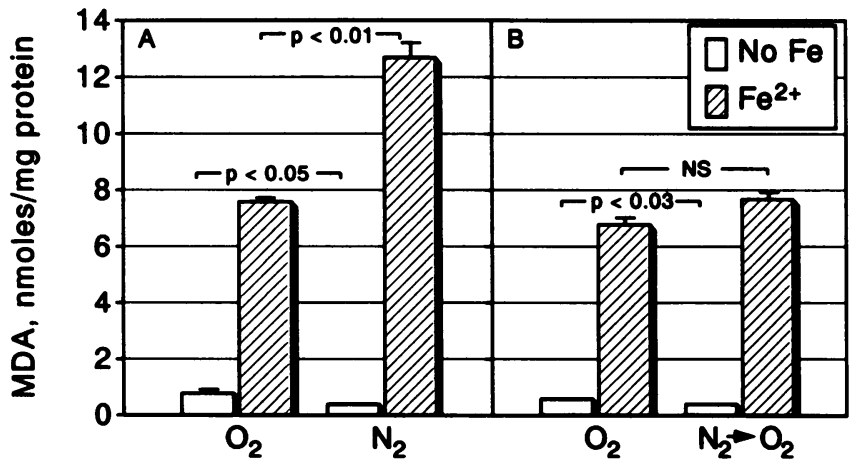

Figure 2. (A) MDA concentrations after $27 \mathrm{~min}$ of oxygenated $\left(\mathrm{O}_{2}\right)$ or hypoxic $\left(\mathrm{N}_{2}\right)$ incubations in the presence or absence of $\mathrm{Fe}^{2+}$. In the absence of $\mathrm{Fe}^{2+}$, hypoxia decreased MDA $(P<0.05$ vs. oxygenated controls). $\mathrm{Fe}^{2+}$ caused a dramatic MDA increment, the degree of which was almost doubled by hypoxic compared with oxygenated incubation $(P<0.01)$. (B) MDA values after $42 \mathrm{~min}$ of oxygenation $\left(\mathrm{O}_{2}\right)$ or after $27 \mathrm{~min}$ of hypoxia plus $15 \mathrm{~min}$ of reoxygenation $\left(\mathrm{N}_{2} \rightarrow\right.$ $\mathrm{O}_{2}$ ) in the presence or absence of $\mathrm{Fe}^{2+}$. Hypoxia-reoxygenation (no $\mathrm{Fe}^{2+}$ ) caused significantly less MDA generation than did continuous oxygenated incubation $(P<0.03)$. $\mathrm{Fe}^{2+}$ caused comparable MDA elevations whether subjected to the $\mathrm{O}_{2}$ or $\mathrm{N}_{2} \rightarrow \mathrm{O}_{2}$ incubations. (Thus, the hypoxia-induced increment in $\mathrm{Fe}^{2+}$-mediated lipid peroxidation $[A]$ was eliminated during reoxygenation, suggesting lipid peroxide metabolism during reoxygenation.) 

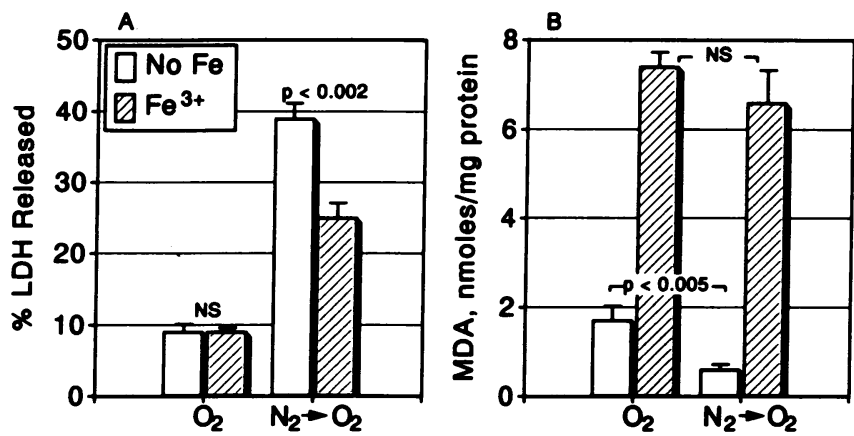

Figure 3. $(A)$ Percent LDH released after either $42 \mathrm{~min}$ of oxygenated incubation $\left(\mathrm{O}_{2}\right)$ or $27 \mathrm{~min}$ of hypoxia $+15 \mathrm{~min}$ of reoxygenation $\left(\mathrm{N}_{2} \rightarrow \mathrm{O}_{2}\right)$ in the presence or absence of $\mathrm{Fe}^{3+} . \mathrm{Fe}^{3+}$ caused no LDH release and it significantly decreased the LDH release caused by the hypoxia-roxygenation protocol. $(B)$ MDA generated by the above protocol. The $\mathrm{N}_{2} \rightarrow \mathrm{O}_{2}$ protocol (no $\mathrm{Fe}^{3+}$ ) decreased MDA production compared with continuous oxygenation $(P<0.005)$. However, the $\mathrm{Fe}^{3+}$-driven MDA increments did not significantly differ (analogous to the Fig. $2 B$ results).

could be determined and it was approximately halved by the $\mathrm{Fe}^{3+}$ addition ( $30 \pm 2$ vs. $16 \pm 2 \% ; P<0.005$ ).

MDA concentrations for these experiments are depicted in Fig. $3 B$. Confirming the observations shown in Fig. $2 B$, hypoxia-reoxygenation in the absence of iron significantly decreased MDA concentrations compared with continuously oxygenated controls $(P<0.005)$. $\mathrm{Fe}^{3+}$ induced marked lipid peroxidation, despite the fact that it induced no cytotoxicity and protected against hypoxia-reoxygenation. Hypoxia-reoxygenation did not alter the iron-driven MDA increments, determined upon the completion of the 42-min experiments (consistent with Fig. $2 B$ ).

Iron effects on PTS adenine nucleotide profiles in the presence and absence of hypoxia. Fig. $4 \mathrm{~A}$ depicts the \%LDH released from the PTS aliquots used for analyzing adenylate pro-

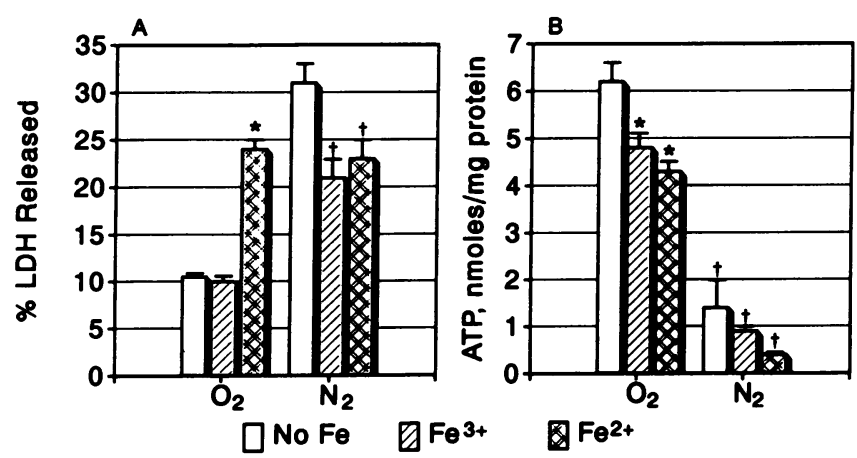

Figure 4. $(A)$ Depiction of LDH release from those PTSs used for adenine nucleotide analysis. As noted previously, $\mathrm{Fe}^{2+}$ in the presence of $\mathrm{O}_{2}$ caused significant $\mathrm{LDH}$ release compared with the control and $\mathrm{Fe}^{3+}$ exposed aliquots $\left(^{*}=P<0.002\right)$. Both $\mathrm{Fe}^{2+}$ and $\mathrm{Fe}^{3+}$ blunted hypoxic $\left(\mathrm{N}_{2}\right) \mathrm{LDH}$ release and approximately to the same degree ( $\dagger=P<0.01$ vs. $\mathrm{N}_{2} /$ no iron exposure). ( $B$ ) ATP concentrations in the above described PTS aliquots. Under oxygenated conditions, $\mathrm{Fe}^{2+}$ and $\mathrm{Fe}^{3+}$ each lowered ATP concentrations $\left({ }^{*}=P<0.01\right.$ vs. oxygenated controls). Despite the fact that $\mathrm{Fe}^{2+}$ and $\mathrm{Fe}^{3+}$ each blunted hypoxic LDH release, each worsened the extent of ATP depletion ( $\dagger=$ all three significantly different, $P<0.05$ ). files. After $27 \mathrm{~min}$ of oxygenated incubation, $\mathrm{Fe}^{2+}$, but not $\mathrm{Fe}^{3+}$, caused significant cytotoxicity. Hypoxic incubation caused $31 \pm 2 \% \mathrm{LDH}$ release. Both $\mathrm{Fe}^{2+}$ and $\mathrm{Fe}^{3+}$ mitigated this injury and approximately to the same extent $(23 \pm 2$ and $21 \pm 3 \%$ LDH release, respectively; both $P<0.001$ vs. hypoxia alone).

Under oxygenated conditions, both $\mathrm{Fe}^{2+}$ and $\mathrm{Fe}^{3+}$ induced $\sim 25 \%$ ATP decrements compared with the non-iron-exposed controls (Fig. $4 \mathrm{~B}$ ). $\mathrm{Fe}^{2+}$ and $\mathrm{Fe}^{3+}$ also significantly worsened hypoxia-induced ATP depletion (ATP concentrations: hypoxia, 1.4 \pm 0.06 ; hypoxia $+\mathrm{Fe}^{3+}, 0.9 \pm 0.09$; hypoxia $+\mathrm{Fe}^{2+}$, $0.4 \pm 0.04 \mathrm{nmol} / \mathrm{mg}$ protein; all significantly different) despite the fact that $\mathrm{Fe}^{2+}$ and $\mathrm{Fe}^{3+}$ each conferred cytoprotection, as noted above. The loss of ATP in these experiments was also expressed in decrements of the total adenine nucleotide pool $\left(\mathrm{ATP}+\mathrm{ADP}+\mathrm{AMP}\right.$ : controls, 7.39 $\pm 0.52 ; \mathrm{Fe}^{2+} / \mathrm{O}_{2}$, $5.67 \pm 0.26 ; \mathrm{Fe}^{3+} / \mathrm{O}_{2}, 5.86 \pm 0.31 ; \mathrm{N}_{2}$ alone, $2.62 \pm 0.16 ; \mathrm{N}_{2} /$ $\left.\mathrm{Fe}^{2+}, 2.22 \pm 0.19 ; \mathrm{N}_{2} / \mathrm{Fe}^{3+}, 2.19 \pm 0.19\right)$.

Effect of $\mathrm{Fe} / \mathrm{DFO}$ on hypoxic-reoxygenation injury. $\mathrm{Fe}^{2+}$ + DFO conferred protection against the hypoxic-reoxygenation protocol (hypoxia-reoxygenation alone, $38 \pm 2 \% \mathrm{LDH}$ release; hypoxia-reoxygenation $+\mathrm{Fe}^{2+}+\mathrm{DFO}, 23 \pm 3 \% ; P$ $<0.002)$. Of note, this protection could not be attributed to DFO alone since it by itself did not alter the hypoxic-reoxygenation injury ( $37 \pm 2 \% \mathrm{LDH}$ release). The DFO addition largely but not completely blocked the iron-mediated MDA increments $\left[\mathrm{Fe}^{2+}\right.$ alone, 7.6 \pm 0.1 (from Fig. 2); $\mathrm{Fe}+\mathrm{DFO}, 1.0 \pm 0.1$; DFO alone, 0.5 \pm 0.06 ; no addition, $0.6 \pm 0.07$ ].

$\mathrm{Fe}^{3+}$ effects on antimycin A-mediated injury. As depicted in Fig. 5, antimycin A induced significant but variable cytotoxicity (overall, $41 \pm 4 \% \mathrm{LDH}$ release; individual values of 31,36 , 39,46 , and $51 \%$ ). $\mathrm{Fe}^{3+}$ addition mitigated this injury in every instance (to $17,23,21,32$, and $33 \%$, respectively, overall $25 \pm 3 \% ; P<0.001)$. This protective effect occurred despite marked $\mathrm{Fe}^{3+}$-mediated lipid peroxidation (MDA values: antimycin $+\mathrm{Fe}^{3+}, 5.8 \pm 0.5$; antimycin alone, $\left.0.7 \pm 0.1\right)$. As with
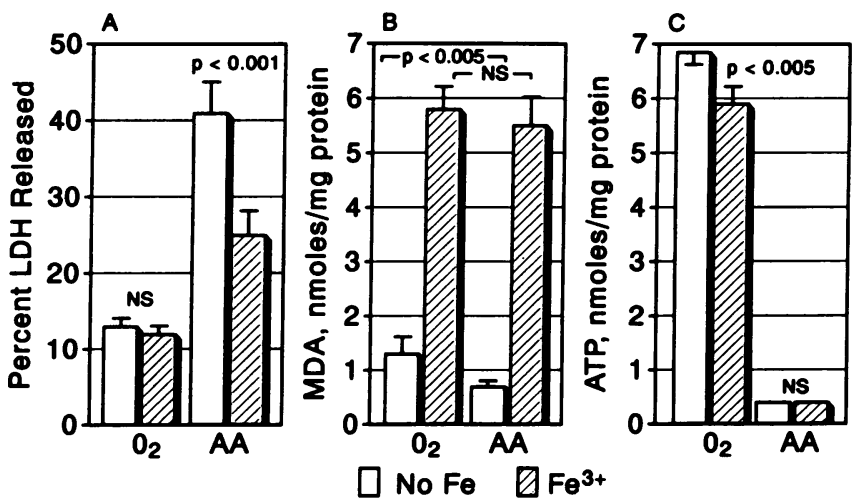

Figure 5. (A) LDH release under oxygenated conditions \pm antimycin A $(A A)$, assessed after 45 min of incubation. $\mathrm{Fe}^{3+}$ substantially decreased AA-induced LDH release $(P<0.001)$. (If one subtracts out the background $\mathrm{LDH}$ release [i.e., in the absence of $\mathrm{AA}$ ], then $\mathrm{Fe}^{3+}$ decreased AA cell killing by $\sim 50 \%$.) (B) MDA concentrations from the above experiments. AA significantly decreased MDA in the absence of $\mathrm{Fe}^{3+}(P<0.005)$. However, AA did not alter the iron-driven MDA increments. $(C)$ ATP concentrations in the above experiments. $\mathrm{Fe}^{3+}$ caused a modest decrease in ATP in the absence of AA $(P<0.005)$. Profound ATP depletion was induced by AA, the degree of which was not altered by concomitant $\mathrm{Fe}^{3+}$ exposure. 
hypoxia, antimycin $\mathrm{A}$ in the absence of iron approximately halved MDA concentrations. However, unlike the hypoxia experiments, antimycin did not increase MDA concentrations in the presence of iron. Antimycin induced profound ATP depletion (from $6.85 \pm 0.2$ to $0.37 \pm 0.01 \mathrm{nmol} / \mathrm{mg}$ protein), a result that was not altered by $\mathrm{Fe}^{3+}$ addition $(0.36 \pm 0.01)$ (indicating that iron did not block the bioavailability of antimycin).

\section{Discussion}

It has been widely proposed that oxygen free radical formation (e.g., from xanthine oxidase activity) with resultant oxidant tissue stress is a critical mediator of ischemic-reperfusion injury of multiple organs $(16,22,23)$, including the kidney (e.g., reference 24 ). It has also been suggested that free (nonproteinbound) iron can dramatically accentuate this pathway of injury, both by directly participating in free radical formation (e.g., ferryl/perferryl ion ), and by catalyzing the conversion of $\mathrm{H}_{2} \mathrm{O}_{2}$ to highly toxic hydroxyl radical via the Fenton/HaberWeiss reactions $(11,16,25-29)$. Recent experiments from this laboratory illustrate this latter point. When BSA was incubated with either $\mathrm{FeSO}_{4}$ or $\mathrm{H}_{2} \mathrm{O}_{2}$, modest protein oxidation was induced (denoted by $\sim 35 \%$ increments in the carbonyl content of BSA ) (10). However, when BSA was exposed to $\mathrm{H}_{2} \mathrm{O}_{2}$ and $\mathrm{Fe}^{2+}$ simultaneously, a 6,000\% carbonyl content increment resulted (unpublished data). Thus, it is clear that iron can indeed dramatically potentiate oxidative tissue reactions. A recent study by Paller and Hedlund (11) suggests that a similar phenomenon may occur in the in vivo kidney. By studying the renal arterial occlusion model of ARF, they observed that $\mathrm{FeCl}_{3}$-EDTA infusion worsened, whereas DFO administration protected against, ischemic injury (11). That $\mathrm{FeCl}_{3}$ increased and DFO decreased renal cortical lipid peroxidation (assessed by MDA) suggested that oxidant mechanisms were operative. The potential relevance of these in vivo observations extends beyond the renal artery occlusion model of ARF. For example, during the initiation phase of myohemoglobinuric ARF, catalytic (free) iron is generated (10), it gains access to tubular lumina (10), and concomitant renal ischemia develops $(8$, 30). Thus, the goal of the present study was to directly assess at the cellular level the impact of catalytic iron on $\mathrm{O}_{2}$ deprivationreoxygenation PTS injury in an effort to better understand mechanisms of myohemoglobinuric as well as ischemic ARF.

To begin these studies, PTSs were subjected to $27 \mathrm{~min}$ of hypoxia plus $15 \mathrm{~min}$ of reoxygenation either in the presence or absence of a toxic $\mathrm{Fe}^{2+}$ concentration. A relatively short hypoxic insult was chosen such that a sufficient number of tubular cells would remain viable, thereby permitting additive/synergistic injury to occur. Despite the fact that hypoxia-reoxygenation alone and $\mathrm{Fe}^{2+}$ alone each induced substantial cell killing ( 31 and $36 \%$ LDH release, respectively), the two in combination produced only $41 \% \mathrm{LDH}$ release (Fig. $1 \mathrm{~A}$ ), suggesting mutually exclusive, not additive/synergistic injury. The simplest possible explanation for this observation would be a "ceiling effect," i.e., that only $41 \%$ of cells could be killed, and thus, synergistic injury could not occur. However, both past experience with the PTS model $(31,32)$ and our present confirmation of $>60 \% \mathrm{LDH}$ release in response to $50 \mathrm{~min}$ of hypoxia exclude this possibility. Thus, the above data indicate that $\mathrm{Fe}^{2+}$ and hypoxia-reoxygenation either induce injury via a common, saturable pathway or that one insult induces cellular changes that confer protection against the other.
In an attempt to gain insights into this issue, the impact of $\mathrm{Fe}^{2+}$ on both the hypoxic and the reoxygenation injury phases were individually assessed. It was reasoned that if the time sequence of injury could be better defined a clearer understanding of why $\mathrm{Fe}^{2+}$ and hypoxia-reoxygenation fail to induce additive injury might result. These experiments indicated the following: $\mathrm{Fe}^{2+}$ significantly blunted the cell injury that resulted from 27 min of hypoxic incubation; this protective effect occurred despite the fact that $27 \mathrm{~min}$ was sufficient time for $\mathrm{Fe}^{2+}$, under oxygenated conditions, to exert its own cytotoxic effect (Fig. $1 B$ ); during reoxygenation $\mathrm{Fe}^{2+}$ exposed/previously hypoxic PTS experienced a rapid phase of LDH release (increase of $17 \%$ ); and this latter phenomenon was of sufficient magnitude to cancel out the previous protective influence of iron. Because it is widely held that iron promotes "reperfusion" injury, it is tempting to postulate that brisk LDH release during reoxygenation was due to synergistic oxidant tissue stress. However, it is important to note that this $17 \% \mathrm{LDH}$ release exactly equalled the sum of independent $\mathrm{Fe}^{2+}(12 \%)$ and reoxygenation $(5 \%)$ effects (defined by the time-matched single insult PTS aliquots). This fact, plus falling rather than rising MDA concentrations during this period (Fig. 2), strongly suggest that this reoxygenation phase was not one of synergistic oxidant tissue injury. In summary, these time frame experiments indicate that, overall, $\mathrm{Fe}^{2+}$ and hypoxia-reoxygenation do not induce fully additive or synergistic injury because $\mathrm{Fe}^{2+}$ mitigates the hypoxic injury phase.

Although the above experiments demonstrated that $\mathrm{Fe}^{2+}$ and hypoxia-reoxygenation do not necessarily cause additive/ synergistic injury, the conclusion that $\mathrm{Fe}^{2+}$ actually mitigates $\mathrm{O}_{2}$ deprivation injury might be questioned because the observed protection was only transient, not extending beyond the hypoxic period. Thus, in an attempt to substantiate that iron can indeed confer significant and durable protection, additional experiments were undertaken using $\mathrm{Fe}^{3+}$ as the iron challenge. The rationale for this was that since $\mathrm{Fe}^{3+}$ has no independent cytotoxic effect it, like $\mathrm{Fe}^{2+}$, might be able to blunt hypoxic injury but it would not induce cell killing during the reoxygenation period. In addition, the ability of $\mathrm{Fe}^{3+}$ to block antimycin A-mediated injury was assessed, both to further substantiate the protective influence of iron and to discern whether this protection can be expressed in the presence of $\mathrm{O}_{2}$, which theoretically permits maximal free radical formation rates. These experiments yielded the following significant results: $\mathrm{Fe}^{3+}$ approximately halved hypoxic injury, whether assessed at the end of hypoxia or after the reoxygenation phase (indicating durable protection); $\mathrm{Fe}^{3+}$ induced its cytoprotective influence despite evoking marked MDA generation; and $\mathrm{Fe}^{3+}$ markedly attenuated antimycin A-induced cell killing, thereby substantiating its protective influence and proving that it can express itself despite high $\mathrm{O}_{2}$ tensions.

Although the above findings seemingly challenge the prevailing view that catalytic iron enhances "ischemic-reperfusion" injury (e.g., references 22-26), a number of potentially important caveats need to be borne in mind before extrapolating these results to the in vivo situation. First, a variety of different mechanisms may be operative in "hypoxic-reoxygenation" vs. "ischemic-reperfusion" injury. Second, although isolated PTSs are widely used as an in vitro model for acute tubular injury, their relevance to in vivo ARF remains to be proven; and third, to induce cell injury and lipid peroxidation in this in vitro system, millimolar ion concentrations were re- 
quired, presumably because of limited iron uptake by tubular cells. Thus, results obtained with these large iron challenges may have limited pathophysiological relevance. However, recent experiments from this laboratory suggest that this concentration issue may not in fact be critical. It was observed that when PTSs were prepared without DFO addition to the collagenase digestion buffer, $\sim 2-10 \mu \mathrm{M}$ PTS iron loading results (32). When such PTSs were incubated under oxygenated conditions, they had limited viability because of the resulting iron toxicity (33 $\pm 1 \% \mathrm{LDH}$ over $60 \mathrm{~min}$ ) (19). However, when these iron-damaged PTSs were challenged with 45 min of hypoxia +15 min of reoxygenation, minimal additional cell killing occurred ( $11 \%$ increment over their oxygenated counterparts vs. a $46 \%$ increment in non-iron-loaded PTS) (19). Thus, these results (which provided the impetus for the current study) strongly support the conclusion that iron, even in micromolar concentrations, can blunt $\mathrm{O}_{2}$ deprivation-mediated PTS injury.

Having confirmed that $\mathrm{Fe}^{2+}$ and $\mathrm{Fe}^{3+}$ can each mitigate hypoxic renal injury, we next addressed whether this protection was mediated via an improvement in cellular energetics. Thus, $\mathrm{Fe}^{2+} / \mathrm{Fe}^{3+}$ effects on adenine nucleotide pools were assessed in the presence and absence of hypoxia. Despite the fact that both iron moieties attenuated hypoxic LDH release, they each exacerbated rather than improved the extent of ATP depletion. Thus, iron protected against hypoxia by mitigating the consequences of ATP depletion rather than the severity of it. That $\mathrm{Fe}^{3+}$ also lessened antimycin A toxicity without improving adenylate pools further supports this conclusion. Of additional interest were findings that $\mathrm{Fe}^{2+}$ and $\mathrm{Fe}^{3+}$ each caused $\sim 25 \%$ decrements in ATP and total adenine nucleotide concentrations under oxygenated conditions. Thus, these data support previous in vivo results suggesting that iron lowers cortical ATP concentrations by $\sim 25 \%$ independent of changes in renal hemodynamics (18). The mechanism for this iron-mediated adenylate loss remains unknown. However, that iron is capable of directly chelating adenine nucleotides (27) and that cell membrane lipid peroxidation might secondarily cause adenine nucleotide depletion are two possibilities. Given that iron causes ATP decrements in the absence of hypoxia, it is not surprising that the degree of ATP depletion during hypoxia was also exacerbated by iron exposure.

The final goal of this study was to assess the impact hypoxia-reoxygenation on proximal tubular lipid peroxidation both in the presence and absence of catalytic iron. Specifically, the following two questions were addressed: does hypoxiareoxygenation cause MDA generation, suggesting a period of rapid free radical formation? and what impact does hypoxiareoxygenation have on iron-driven MDA increments? As depicted in Fig. 2, hypoxia not unexpectedly decreased MDA generation by non-iron-exposed PTS, suggesting that $\mathrm{O}_{2}$ deprivation limits oxygen free radical formation. However, during reoxygenation, no rebound lipid peroxidation resulted, MDA concentrations remaining significantly lower than those found in the continuously oxygenated controls. Thus, these data support a growing body of evidence that indicates that $\mathrm{O}_{2}$ deprivation-reoxygenation PTS injury is not oxygen free radical/lipid peroxidation dependent (19, 32-34). Paradoxically, hypoxia appeared to double the amount of iron-mediated lipid peroxidation, at least as assessed by the MDA assay. The reason for this surprising result is unknown. Since MDA levels undoubtedly reflect a balance between lipid peroxide synthesis and mi- tochondrial metabolism, one possible explanation is that sufficient $\mathrm{O}_{2}$ is present during hypoxia to permit the former, but not the latter, to occur. However, that antimycin A did not also induce an increment in iron-driven MDA generation speaks against this possibility. Thus, an alternative explanation for the greater MDA increments is that $\mathrm{Fe}^{2+}$ and hypoxia induce synergistic oxidant stress via the Fenton/Haber-Weiss reactions. However, if true, it is important to note that this temporally correlated with cytoprotection, not cell injury. Conversely, during reoxygenation MDA concentrations fell (presumably due to lipid peroxide catabolism) despite the fact that cellular injury resulted. Thus, these results serve to underscore our previous conclusions (15) that MDA generation and critical cell injury may bear little relationship.

The mechanism by which iron confers protection against hypoxic- and antimycin-mediated injury remains to be defined. One intriguing possibility is that iron-induced lipid peroxidation, rather than being injurious, might paradoxically confer a protective effect. To test this possibility, the influence of iron + DFO on hypoxia-reoxygenation injury was assessed. Since excess DFO can block iron-mediated lipid peroxidation, if iron + DFO were able to confer protection, it would serve to dissociate iron-induced lipid peroxidation from its cytoprotective effect. This appeared to be the case since iron + DFO substantially reduced hypoxic-reoxygenation injury despite trivial MDA generation. Of note, this cytoprotective influence cannot be explained by a pure DFO effect since both past (32, 34 ) and present data indicate that DFO does not by itself protect PTS from hypoxic-reoxygenation injury.

Lastly, although the present experiments were directed at exploring the nature of iron-hypoxic interactions, they also provide two new insights into determinants of $\mathrm{Fe}^{2+}$-induced cytotoxicity. First, that $\mathrm{Fe}^{2+}$ appeared to cause no $\mathrm{LDH}$ release under hypoxic conditions (Fig. 4) indicates that $\mathrm{Fe}^{2+}$ cytotoxicity is $\mathrm{O}_{2}$ dependent. Although this suggests that $\mathrm{Fe}^{2+}$ toxicity is due to an oxygen free radical-dependent reaction, the finding of greater $\mathrm{Fe}^{2+}$-mediated MDA elevations during $\mathrm{Fe}^{2+} /$ hypoxic incubation versus $\mathrm{Fe}^{2+}$ /oxygenated incubation (Fig. $2 \mathrm{~A}$ ) argues against this hypothesis. Thus, an alternative possibility is that hypoxia precludes the expression of $\mathrm{Fe}^{2+}$ toxicity because the resulting ATP depletion prevents $\mathrm{Fe}^{2+}$ transport to critical intracellular targets. Although this remains a hypothesis, it does point out that an $\mathrm{O}_{2}$ requirement for the expression of $\mathrm{Fe}^{2+}$ toxicity does not necessarily equate with a free radicalbased reaction. Second, the present study demonstrates for the first time that $\mathrm{Fe}^{2+}$-induced PTS toxicity is not mediated by ATP depletion. This conclusion is based on findings that 27 min of $\mathrm{Fe}^{2+}$ exposure induced LDH release despite only modest ATP reductions $(\sim 25 \%)$ and that $\mathrm{Fe}^{2+}$ and $\mathrm{Fe}^{3+}$ caused comparable ATP losses and yet only the former caused LDH release.

In conclusion, the present study indicates that cytotoxic $\mathrm{Fe}^{2+}$ can blunt hypoxic PTS injury despite inducing lipid peroxidation and lowering ATP. However, this beneficial effect is transitory because during reoxygenation $\mathrm{Fe}^{2+}$ is able to express its intrinsic cytotoxic effect. $\mathrm{Fe}^{3+}$, like $\mathrm{Fe}^{2+}$, also blunts hypoxic PTS injury. However, unlike $\mathrm{Fe}^{2+}, \mathrm{Fe}^{3+}$-mediated protection persists throughout reoxygenation because it exerts no intrinsic cytotoxicity. $\mathrm{Fe}^{3+}$ also protects against antimycin $\mathrm{A}-$ mediated PTS injury independent of changes in cellular energetics. This confirms that iron can indeed blunt ATP depletion-mediated injury and that this protective influence is not dependent on 
concomitant hypoxia (which theoretically could limit oxygen free radical generation ); and hypoxia and antimycin $\mathrm{A}$ inhibit PTS lipid peroxide formation. However, hypoxia paradoxically increases iron-driven MDA increments, possibly by creating the necessary conditions for the Fenton/Haber-Weiss reactions. In sum, although these experiments indicate that iron may promote lipid peroxidation during hypoxia, cytoprotection rather than additive/synergistic injury can result. Thus, the ability of heme proteins to exacerbate in vivo ischemic tubular injury cannot simply be explained by a direct ironischemic interaction. Previous observations from this laboratory that both iron- and non-iron-containing filtered proteins can equally exacerbate experimental ischemic renal injury further support this view (6).

\section{Acknowledgments}

We thank Ms. D. Jensen for her clerical expertise.

This work was supported by a grant from the National Institutes of Health (DK 38432).

\section{References}

1. Badenoch, A. W., and E. M. Parmady. 1948. The effect of stroma free hemoglobin on the ischaemic kidney of the rabbit. Br. J. Exp. Pathol. 29:215223.

2. Yuile, C. L., M. A. Gold, and E. G. Hinds. 1945. Hemoglobin precipitation in renal tubules. J. Exp. Med. 82:361-374.

3. Conn, H. L., J. C. Wood, and J. C. Rose. 1956. Circulatory and renal effects following transfusion of human blood and its components to dogs. Circ. Res. 4:18-24.

4. Jaenike, J. R. 1967. The renal lesion associated with hemoglobinuria. A study of the pathogenesis of the excretory defect in the rat. J. Clin. Invest. 46:378387.

5. Zager, R. A., and L. M. Gamelin. 1989. Pathogenetic mechanisms in experimental hemoglobinuric acute renal failure. Am. J. Physiol. 256:F446-F455.

6. Zager, R. A., E. J. Teubner, and S. Adler. 1987. Low molecular weight proteinuria exacerbates experimental ischemic renal injury. Lab. Invest. 56:180 188.

7. Zager, R. A., J. Mahan, and A. J. Merola. 1985. Effects of mannitol on the postischemic kidney: biochemical, functional, and morphologic assessments. Lab. Invest. 53:433-442.

8. Zager, R. A., C. A. Foerder, and C. R. Bredl. 1991. The influence of mannitol on myoglobinuric acute renal failure: functional, biochemical, and morphologic assessments. J. Am. Soc. Nephrol. 2:848-855.

9. Zager, R. A. 1989. Studies of mechanisms and protective maneuvers in myoglobinuric acute renal failure. Lab. Invest. 60:619-629.

10. Zager, R. A. 1992. Combined mannitol and deferoxamine therapy for myohemoglobinuric renal injury and oxidant tissue stress: mechanistic and therapeutic implications. J. Clin. Invest. 90:711-719.

11. Paller, M. S., and B. E. Hedlund. 1988. Role of iron in postischemic renal injury in the rat. Kidney Int. 34:474-480.

12. Paller, M. S. 1988. Hemoglobin- and myoglobin-induced acute renal failure in rats: role of iron in nephrotoxicity. Am. J. Physiol. 255:F539-F544.
13. Shah, S. V., and P. D. Walker. 1988. Evidence suggesting a role for hydroxyl radical in glycerol-induced acute renal failure. Am. J. Physiol. 255:F438F443.

14. Corcoran, A. C., and I. H. Page. 1945. Renal damage from ferroheme pigments myoglobin, hemoglobin, hematin. Tex. Rep. Biol. Med. 3:528-544.

15. Zager, R. A., and C. A. Foerder. 1992. Effects of inorganic iron and myoglobin on in vitro proximal tubular lipid peroxidation and cytotoxicity. $J$. Clin. Invest. 89:989-995.

16. Halliwell, B., and J. M. C. Gutteridge. 1991. Role of free radicals and catalytic metal ions in human disease: an overview. Methods Enzymol. 186:1-85.

17. Halliwell, B., and J. M. C. Gutteridge. 1984. Oxygen toxicity, oxygen radicals, transition metals and disease. Biochem. J. 219:1-14.

18. Zager, R. A. 1991. Myoglobin depletes renal adenine nucleotide pools in the presence and absence of shock. Kidney Int. 39:111-119.

19. Zager, R. A., D. J. Gmur, B. A. Schimpf, C. R. Bredl, and C. A. Foerder. 1992. Evidence against increased hydroxyl radical production during oxygen deprivation/reoxygenation proximal tubular injury. J. Am. Soc. Nephrol. 2:16271633.

20. Uchiyama, M., M. Mihara. 1978. Determination of malonaldehyde precursor in tissues by thiobarbituric acid test. Anal. Biochem. 86:271-278.

21. Stocchi, V., L. Cuccharini, T. Canestrari, M. P. Piacentini, and G. Fornaini. 1987. A very fast ion-paired reverse phase HPLC method for separation of the most significant nucleotides and their degradation products in human red blood cells. Anal. Biochem. 167:181-185.

22. McCord, J. M. 1985. Oxygen-derived free radicals in post-ischemic tissue injury. N. Engl. J. Med. 312:159-163.

23. Cross, C. E., B. Halliwell, E. T. Boush, W. A. Pryor, B. N. Amos, R. C. Saul, J. M. McCord, and D. Harman. 1987. Oxygen radicals and human disease. Ann. Intern. Med. 107:526-545.

24. Paller, M. S., J. R. Hoidal, and T. F. Ferris. 1984. Oxygen free radicals in ischemic acute renal failure in the rat. J. Clin. Invest. 74:1156-1164.

25. Williams, R. E., J. L. Zweier, and J. T. Flaherty, 1991. Treatment with deferoxamine during ischemia improves functional and metabolic recovery and reduces reperfusion-induced oxygen radical generation in rabbit hearts. Circulation. 83:1006-1014.

26. Smith, J. K., D. L. Carden, M. B. Grisham, D. N. Granger, and R. J. Korthuis. 1989. Role of iron in postischemic microvascular injury. Am. J. Phys iol. 256:H1472-H1477.

27. Svingen, B. A., F. O. O'Neil, and S. D. Aust. 1978. The role of superoxide and singlet oxygen in lipid peroxidation. Photochem. Photobiol. 28:803-809.

28. Rush, J. D., and W. H. Koppenol. 1986. Oxidizing intermediates in the reaction of ferrous EDTA with hydrogen peroxide: reactions with organic molecules and ferrocytochrome C. J. Biol. Chem. 261:6730-6733.

29. Mitsos, E., D. Kim, B. R. Lucchesi, and J. C. Fantone. 1988. Modulation of myoglobin- $\mathrm{H}_{2} \mathrm{O}_{2}$ mediated peroxidative reactions by sulfhydryl compounds. Lab. Invest. 59:824-830.

30. Odeh, M. 1991. The role of reperfusion-induced injury in the pathogenesis of the crush syndrome. N. Engl. J. Med. 324:1417-1422.

31. Zager, R. A., D. J. Gmur, C. R. Bredl, and M. J. Eng. 1991. Temperature effects on ischemic and hypoxic renal proximal tubular injury. Lab. Invest. 64:766-775.

32. Zager, R. A., B. A. Schimpf, C. R. Bredl, and C. A. Foerder. 1992. In creased proximal tubular cell catalytic iron content: a result, not a mediator of, hypoxic-reoxygenation injury. J. Am. Soc. Nephrol. 3:116-118.

33. Doctor, R. B., and L. J. Mandel. 1991. Minimal role of xanthine oxidase and oxygen free radicals in rat renal tubular reoxygenation injury. J. Am. Soc. Nephrol. 1:959-969.

34. Weinberg, J. M., J. A. Davis, M. Abarzua, and T. Rajan. 1987. Cytoprotective effects of glycine and glutathione against hypoxic injury to renal tubules J. Clin. Invest. 80:1446-1454. 